\title{
Simplified quantum bit commitment using single photon nonlocality
}

\author{
Guang Ping $\mathrm{H}^{\text {* }}$ \\ School of Physics and Engineering, Sun Yat-sen University, Guangzhou 510275, China
}

\begin{abstract}
We simplified our previously proposed quantum bit commitment (QBC) protocol based on the Mach-Zehnder interferometer, by replacing symmetric beam splitters with asymmetric ones. It eliminates the need for random sending time of the photons; thus, the feasibility and efficiency are both improved. The protocol is immune to the cheating strategy in the Mayers-Lo-Chau no-go theorem of unconditionally secure QBC, because the density matrices of the committed states do not satisfy a crucial condition on which the no-go theorem holds.
\end{abstract}

PACS numbers: 03.67.Dd, 03.67.Hk, 42.50.Ex, 03.65.Ud, 42.50.St, 03.67.Ac

\section{INTRODUCTION}

Quantum bit commitment (QBC) [1, 2] is a two-party cryptography including two phases. In the commit phase, Alice (the sender of the commitment) decides the value of the bit $b(b=0$ or 1$)$ that she wants to commit, and sends Bob (the receiver of the commitment) a piece of evidence, e.g., some quantum states. Later, in the unveil phase, Alice announces the value of $b$, and Bob checks it with the evidence. An unconditionally secure QBC protocol needs to be both binding (i.e., Alice cannot change the value of $b$ after the commit phase) and concealing (Bob cannot know $b$ before the unveil phase) without relying on any computational assumption.

QBC is recognized as an essential primitive for quantum cryptography, as it is the building block for quantum multi-party secure computations and more complicated "post-cold-war era" multi-party cryptographic protocols 3, 4]. Unfortunately, it is widely accepted that unconditionally secure QBC is impossible [5]-28], despite of some attempts toward secure ones (e.g., 29 34] and the references therein). This result, known as the Mayers-LoChau (MLC) no-go theorem, was considered as putting a serious drawback on quantum cryptography. (Note that cheat-sensitive QBC [35 37] is not covered, as its security goal is defined differently from that of the QBC studied in the current paper.)

Very recently, we proposed a $\mathrm{QBC}$ protocol using orthogonal states [29], where the density matrices do not satisfy a crucial condition on which the MLC no-go theorem holds. The protocol is based on the GoldenbergVaidman (GV) quantum key distribution (QKD) scheme 38], which makes use of the Mach-Zehnder interferometer involving symmetric beam splitters. Koashi and Imoto [39] pointed out that the GV QKD scheme can be simplified by replacing the symmetric beam splitters with asymmetric ones. Here we will apply the same idea to propose a simplified version of our QBC protocol, so that it can be more feasible and efficient.

In the next section, we introduce some basic notations

*Electronic address: hegp@mail.sysu.edu.cn and settings used throughout the paper. The KoashiImoto (KI) QKD scheme will be briefly reviewed in section III. Then we propose our QBC protocol in section IV, and analyze its security in section V. The feasibility will be discussed in section VI. Section VII summarizes the conclusion and gives some remarks. An example of the technical attack mentioned in section VI will be provided in the appendix.

\section{NOTATIONS AND SETTINGS}

Generally, in both QKD and QBC the two participants are called Alice and Bob. But similar to [29], in our current $\mathrm{QBC}$ protocol, the behavior of Bob is more like that of the eavesdropper rather than the Bob in QKD. To avoid confusion, here we use the names in the following way. In $\mathrm{QKD}$, the sender of the secret information is called Alice, the receiver is renamed as Charlie instead of Bob, and the external eavesdropper is called Eve. In QBC, the sender of the commitment is Alice, the receiver is Bob, and there is no Eve since QBC merely deals with the cheating from internal dishonest participants, instead of external eavesdropping.

As our main interest is focused on the theoretical possibility of secure QBC, we will only consider the ideal case where no transmission error occurs in the communication channels, nor there are detection loss or dark counts, etc.

\section{THE KOASHI-IMOTO QKD SCHEME}

Our QBC proposal is inspired by the KI QKD scheme [39], which makes use of the Mach-Zehnder interferometer illustrated in FIG. 1. Let $R$ and $T$ denote the reflectivity and transmissivity of the asymmetric beam splitters $B S_{1}$ and $B S_{2}$, with $R+T=1$ and $R \neq T$. Alice encodes the bit values 0 and 1 she wants to transmit to Charlie, respectively, using two orthogonal states

$$
\begin{aligned}
& 0 \rightarrow\left|\Psi_{0}\right\rangle \equiv \sqrt{T}|0\rangle_{X}|1\rangle_{Y}-i \sqrt{R}|1\rangle_{X}|0\rangle_{Y}, \\
& 1 \rightarrow\left|\Psi_{1}\right\rangle \equiv \sqrt{T}|1\rangle_{X}|0\rangle_{Y}-i \sqrt{R}|0\rangle_{X}|1\rangle_{Y} .
\end{aligned}
$$

Here $|m\rangle_{j}$ is the $m$ photon Fock state for the arm $j=$ $X, Y$. That is, each $\left|\Psi_{0}\right\rangle$ or $\left|\Psi_{1}\right\rangle$ is split into two localized 


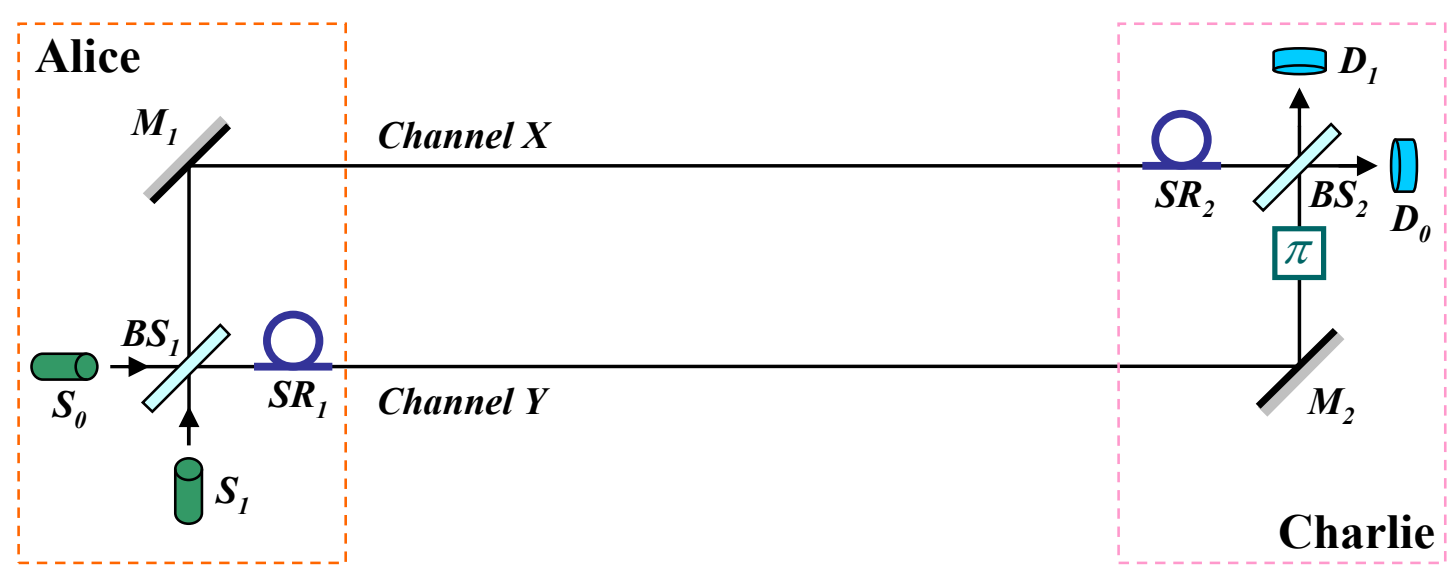

FIG. 1: Diagram of the experimental implementation of the Koashi-Imoto QKD scheme 39]. The state of a photon produced by the source $S_{0}\left(S_{1}\right)$ will become $\left|\Psi_{0}\right\rangle=\sqrt{T}|0\rangle_{X}|1\rangle_{Y}-i \sqrt{R}|1\rangle_{X}|0\rangle_{Y}\left(\left|\Psi_{1}\right\rangle=\sqrt{T}|1\rangle_{X}|0\rangle_{Y}-i \sqrt{R}|0\rangle_{X}|1\rangle_{Y}\right)$ after passing the asymmetric beam splitter $B S_{1}$ with reflectivity $R$ and transmissivity $T$. The two wave packets of the same photon are sent through channels $X$ and $Y$ respectively. When no eavesdropper presented, the storage rings $S R_{1}, S R_{2}$, the mirrors $M_{1}, M_{2}$ and the phase shifter $\pi$ will ensure the complete apparatus work as a Mach-Zehnder interferometer, so that $\left|\Psi_{0}\right\rangle\left(\left|\Psi_{1}\right\rangle\right)$ will be detected by the detector $D_{0}\left(D_{1}\right)$ with certainty.

wave packets, and sent to Charlie separately in quantum channels $X$ and $Y$, respectively; thus single photon nonlocality is presented. This is done by sending a single photon either from the source $S_{0}$ (sending $\left|\Psi_{0}\right\rangle$ ) or from the source $S_{1}$ (sending $\left|\Psi_{1}\right\rangle$ ), then splitting it with the beam splitter $B S_{1}$ made of a half-silvered mirror (note that polarizing beam splitters are not recommended due to the security problem addressed at the end of section 6 of $[29]$ ).

To ensure the security of the transmission, the wave packet in channel $Y$ is delayed by the storage ring $S R_{1}$, which introduces a sufficiently long delay time $\tau$ so that this wave packet will not leave Alice's site until the other wave packet in channel $X$ already entered Charlie's site. Thus the two wave packets of the same photon are never present together in the transmission channels. This makes it impossible for Eve to prepare and send Charlie a perfect clone on time if she waits to intercept and measure both wave packets, even though $\left|\Psi_{0}\right\rangle$ and $\left|\Psi_{1}\right\rangle$ are orthogonal, because she has to decide what to resend to channel $X$ before she can receive anything from channel $Y$. On the other hand, when no eavesdropping occurs, Charlie can distinguish $\left|\Psi_{0}\right\rangle$ and $\left|\Psi_{1}\right\rangle$ unambiguously by adding a storage ring $S R_{2}$ to channel $X$ whose delay time is also $\tau$, while introducing a phase shift $\pi$ to channel $Y$. The two wave packets of the same photon will then recombine and interfere on the beam splitter $B S_{2}$, which is identical to $B S_{1}$. Thus the complete apparatus of Alice and Charlie forms a Mach-Zehnder interferometer, so that $\left|\Psi_{0}\right\rangle\left(\left|\Psi_{1}\right\rangle\right)$ will always make the detector $D_{0}\left(D_{1}\right)$ click with certainty, allowing Charlie to decode the transmitted bit correctly. Any mismatched result between Alice's transmitted state and Charlie's measurement will immediately reveals the presence of Eve [39].

Comparing with the GV QKD scheme [38], the key difference is that $B S_{1}$ and $B S_{2}$ in the KI scheme are asymmetric beam splitters, while the GV scheme uses symmetric ones. The advantage of this modification is that the sending time of each photon can be fixed and publicly known beforehand, while in the GV scheme it has to be random and kept secret from Eve until the security check.

An important fact that will be useful for our QBC protocol is that, since the KI scheme is unconditionally secure, it is clear that Eve's intercept-resend attack will always be detected with a nontrivial probability. That is, if she intercepts a state sent from Alice and decodes a nontrivial amount of information, then the state she resends to Charlie cannot always make Charlie's correct detector click at the right time with the probability $100 \%$, no matter what is Eve's strategy on preparing the resent state. There will always be a nontrivial probability that the resent state will be detected by the wrong detector (the one that should not click when no eavesdropping presented), or at the wrong time (which is earlier or later than the correct arrival time when the state is not intercepted). Please see [39] for the rigorous proof, as well as some examples showing why Eve's strategies do not work.

\section{OUR QBC PROTOCOL}

As illustrated in FIG. 2, to build a QBC protocol upon the above KI QKD scheme, we treat Charlie's site as a part of Alice's, so that the two parties merge into one. Alice sends out a bit-string $c$ encoded with the above orthogonal states, whose value is related to the bit $b$ she wants to commit. Then she receives the states herself. Meanwhile, let Bob take the role of Eve. His action shifts 


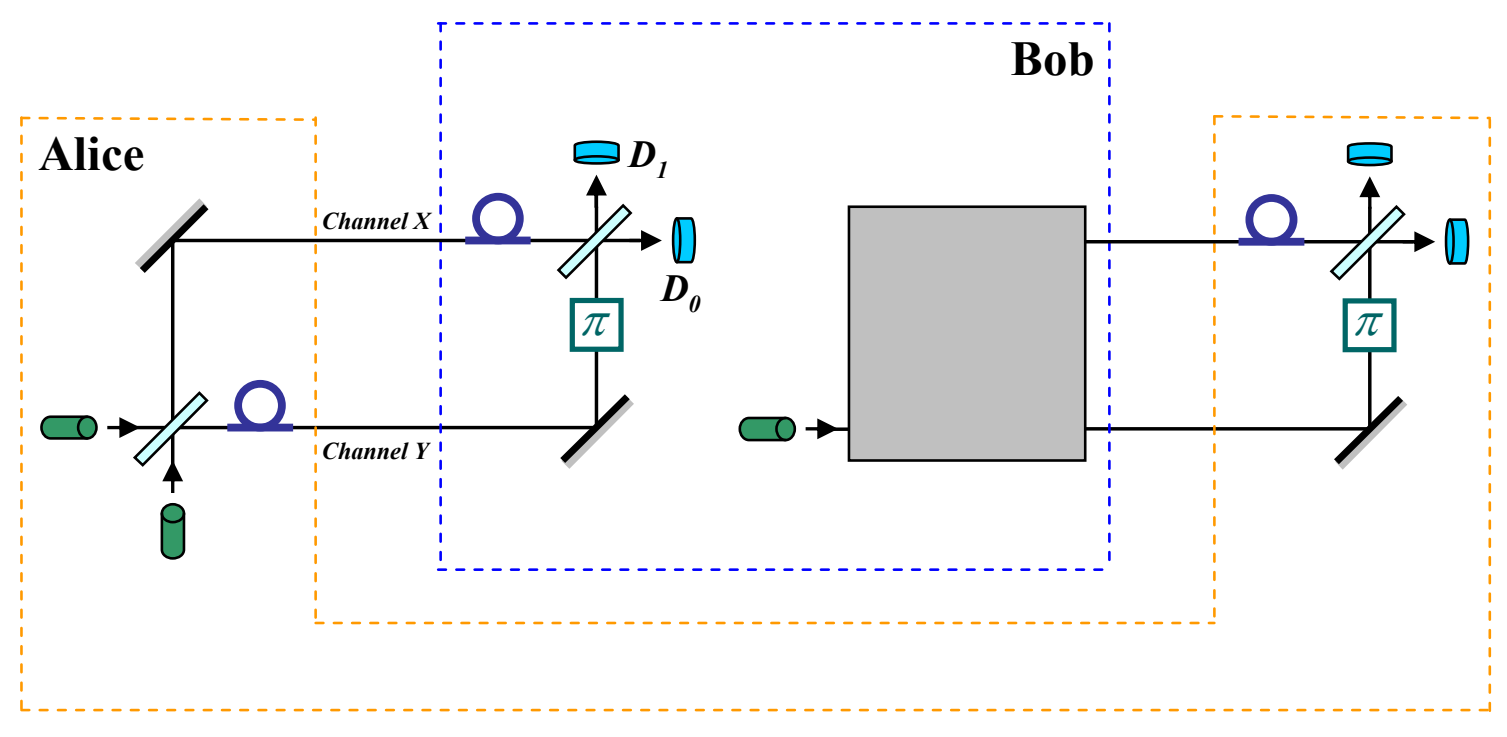

FIG. 2: Diagram for the apparatus of the QBC protocol when Bob chooses the intercept mode. He measures Alice's photon using the same device as that of Alice's, while sending another photon to Alice according to a certain strategy (corresponding to the device illustrated as the black box in the diagram) to reduce Alice's probability of finding his interception.

between two modes. In the bypass mode, he simply does nothing so that the corresponding parts of the states return to Alice intact. In the intercept mode, he applies the intercept-resend attack. That is, he intercepts the state and decodes the corresponding bit (which can be done using the same device as that of Charlie's), and prepares a fake state to resend to Alice on time. While there could be many strategies for Bob to prepare the resent state, we use $\varepsilon$ to denote the lower bound of the average probability for his resent state to be caught in Alice's check. As we mentioned above, the unconditional security of the KI QKD scheme guarantees that $\varepsilon$ cannot always equal exactly to zero for both $\left|\Psi_{0}\right\rangle$ and $\left|\Psi_{1}\right\rangle$ even when Bob uses the optimal strategy. Therefore, Alice is able to estimate the upper bound of the frequency of the presence of the intercept mode, to limit Bob from intercepting the whole string $c$, so that the value of the committed bit $b$ can be made concealing. Meanwhile, since $\varepsilon<1$, at the end of the commit phase there will be some bits of the string become known to Bob, while Alice does not know the exact position of all these bits. Thus she cannot alter string $c$ freely at a later time, making the protocol binding. The complete QBC protocol is described below.

The commit protocol:

(1) Bob chooses a binary linear $(n, k, d)$-code $C$ [2] and announces it to Alice, where $n, k, d$ are agreed on by both Alice and Bob.

(2) Alice chooses a nonzero random $n$-bit string $r=$ $\left(r_{1} r_{2} \ldots r_{n}\right) \in\{0,1\}^{n}$ and announces it to Bob. This makes any $n$-bit codeword $c=\left(c_{1} c_{2} \ldots c_{n}\right)$ in $C$ sorted into either of the two subsets $C_{(0)} \equiv\{c \in C \mid c \odot r=0\}$ and $C_{(1)} \equiv\{c \in C \mid c \odot r=1\}$. Here $c \odot r \equiv \bigoplus_{i=1}^{n} c_{i} \wedge r_{i}$.
(3) Now Alice decides the value of the bit $b$ that she wants to commit. Then she chooses a codeword $c$ from $C_{(b)}$ randomly.

(4) Alice encodes each bit of this specific $c$ as $c_{i} \rightarrow$ $\left|\Psi_{c_{i}}\right\rangle$ where the state $\left|\Psi_{c_{i}}\right\rangle$ is defined by equation (11). She sends Bob the two wave packets of the same state separately in channels $X$ and $Y$, with the storage ring $S R_{1}$ on channel $Y$ introducing a delay time $\tau$ known to Bob.

(5) For each of Alice' states $\left|\Psi_{c_{i}}\right\rangle(i=1, \ldots, n)$, Bob chooses the intercept mode with probability $f(f<1-$ $d / n)$ and the bypass mode with probability $1-f$.

If Bob chooses to apply the bypass mode, he simply keeps channels $X$ and $Y$ intact so that the state sent from Alice will be returned to her detectors as is.

Else if Bob chooses to apply the intercept mode, he uses the same measurement device as that of Alice's, to measure $\left|\Psi_{c_{i}}\right\rangle$ so that he can decode $c_{i}$ with certainty. Meanwhile, he prepares another state and sends it back to channels $X$ and $Y$ at the right time, i.e., the time which can ensure that it reaches Alice's detectors at a time that looks as if Bob were applying the bypass mode.

There are many different strategies how Bob sends this state (thus we left this part of Bob's device as a black box in FIG. 2). One of the simplest ways is to use the same device that Alice uses for sending her state. More strategies will be discussed in section V.A. It will be shown there that all these strategies cannot hurt the security of the protocol, so that Bob are allowed to use any of them here. As stated above, in any strategy there is a nonvanishing probability that Bob's resent state does not equal to Alice's original $\left|\Psi_{c_{i}}\right\rangle$ since he has to send it before completing the measurement on $\left|\Psi_{c_{i}}\right\rangle$ (to be further explained in section V.A too). Let $\varepsilon$ be the lower bound 
of this probability for all these strategies.

(6) Alice uses the same device that Charlie used in the KI QKD scheme, to measure the output of the quantum channels from Bob. She counts how many times her measurement results do not match the states she sent, and denotes it as $n^{\prime}$. From step (5) it can be shown that $n^{\prime} \sim \varepsilon f n$. Thus Alice can estimate the upper bound of the probability of Bob choosing the intercept mode as $f \sim n^{\prime} /(\varepsilon n)$. Alice agrees to continue with the protocol if she finds $f<1-d / n$, which means that the number of $c_{i}$ 's known to Bob is $f n<n-d$. Otherwise she concludes that Bob is cheating.

The unveil protocol:

(7) Alice announces the values of $b$ and the specific $c=\left(c_{1} c_{2} \ldots c_{n}\right)$ she chose.

(8) Bob accepts the commitment if $c \odot r=b$ and $c$ is indeed a codeword from $C$, and every $c_{i}$ agrees with the state $\left|\Psi_{c_{i}}\right\rangle$ he detected in the intercept mode.

\section{SECURITY}

\section{A. Basic ideas}

For easy understanding, here we will first give a heuristic explanation of the security of the protocol. A more general theoretical proof will be provided in section V.B.

The binary linear $(n, k, d)$-code $C$ used in the protocol can simply be viewed as a set of classical $n$-bit strings. Each string is called a codeword. This set of strings has two features.

(A) Among all the $2^{n}$ possible choices of $n$-bit strings, only a particular set of the size $\sim 2^{k}(k<n)$ is selected to form this set.

(B) The distance (i.e., the number of different bits) between any two codewords in this set is not less than $d$ $(d<n)$.

Feature (A) puts a limit on Alice's freedom on choosing the initial state $\left|\psi_{c}\right\rangle \equiv\left|\Psi_{c_{1}}\right\rangle \otimes\left|\Psi_{c_{2}}\right\rangle \otimes \ldots \otimes\left|\Psi_{c_{i}}\right\rangle \otimes$ $\ldots \otimes\left|\Psi_{c_{n}}\right\rangle$, since each $\left|\Psi_{c_{i}}\right\rangle(i=1, \ldots, n)$ cannot be chosen independently. Instead, the string $c=\left(c_{1} c_{2} \ldots c_{n}\right)$ formed by the indices $c_{i}$ 's can only be chosen among the codewords. Meanwhile, feature (B) guarantees that if Alice wants to change the string $c$ from one codeword into another so that the value of her committed $b$ can be altered, she needs to change at least $d$ bits of the codeword $c$. But among the $n$ states she sent to Bob, there are only $n^{\prime} \sim \varepsilon f n$ states which she knows for sure that Bob has applied the intercept mode. For each of the rest $n-n^{\prime}$ states, her measurement result always matches the state she sent, so that she cannot distinguish unambiguously whether Bob has applied the bypass mode or the intercept mode. If it was the intercept mode, Bob already knew the corresponding bit $c_{i}$ of the codeword $c$, so that Alice's altering it will be caught inevitably. Rigorously speaking, among these $n-n^{\prime}$ states, the probability that
Bob has applied the intercept mode is

$$
p=\frac{f n-n^{\prime}}{n-n^{\prime}}=\frac{f-\varepsilon f}{1-\varepsilon f} .
$$

Therefore Alice's altering one bit of the codeword will stand the probability $p$ to be detected, and her probability of altering $\geq d$ bits without being detected will be $(1-p)^{d}$. Alternatively, Alice may prepare $\left|\psi_{c}\right\rangle$ initially in a state where $c$ is not a codeword. Instead, it is half-way between two codewords, so that changing $d / 2$ will be sufficient to turn it into one of the codewords. In this case, her probability of escaping the detection will be increased to $(1-p)^{d / 2}$. Nevertheless, in either way the probability drops exponentially as $d$ increases. Thus it can be made arbitrarily close to zero.

On the other hand, feature (A) also guarantees that the number of different codewords having less than $n-d$ bits in common increases exponentially with $k$. This is the key that makes our protocol secure against Bob, as Alice can bound the frequency $f$ that Bob applies the intercept mode, which in turns bounds the number of the bits known to Bob so that he cannot know Alice's actual choice of the codeword. The reason is that in step (5) of the protocol, no matter what is the strategy that Bob used in his intercept mode to prepare the resent state, it can be shown that his resent state cannot always equal to the $\left|\Psi_{c_{i}}\right\rangle$ he received from Alice, as long as he wants to ensure that the time it reaches Alice's detectors will show no difference than the case where he were applying the bypass mode. That is, either Bob's resent state in the intercept mode will inevitably make Alice's detectors click earlier or later than the time it does when the bypass mode were used instead, or the resent state will be different from $\left|\Psi_{c_{i}}\right\rangle$ with a nonvanishing probability $\varepsilon$.

To see why this is the case, let us first consider the strategy where Bob prepares the resent state using the same device that Alice uses for sending her $\left|\Psi_{c_{i}}\right\rangle$. Suppose that the first wave packet of Alice's $\left|\Psi_{c_{i}}\right\rangle$ enters Bob's site via channel $X$ (before entering his storage ring) at time $t_{i}$ (which should be agreed on by both Alice and Bob beforehand). Then the second wave packet of $\left|\Psi_{c_{i}}\right\rangle$ will enter Bob's site via channel $Y$ at time $t_{i}+\tau$ due to the existence of the storage ring in Alice's sending device (see FIG. 2). As there is also another storage ring in Alice's measurment device, Bob must send the first wave packet of his resent state into channel $X$ at time $t_{i}$, and send the second wave packet into channel $Y$ at time $t_{i}+\tau$, so that they can reach Alice's measurement device at a time that looks like Bob is running the bypass mode. Here for simplicity, we assume that the time for the wave packets to travel the rest part of the channels (other than the storage rings) is negligible. That is, the first wave packet of Bob's state needs to be sent before the second wave packet of Alice's $\left|\Psi_{c_{i}}\right\rangle$ reaches him. Otherwise his resent state will reach Alice's detectors later than the expected arrival time when no interception occurs. Therefore, by the time Bob prepares the resent state, he has not received Alice' state completely yet so he does not know 
the form of $\left|\Psi_{c_{i}}\right\rangle$. Thus his resent state cannot match Alice's state with probability $100 \%$, and he cannot make them match by his local operations acting solely on the second wave packet of his resent state (the one to be sent via channel $Y$ ) after the first wave packet in channel $X$ was already sent.

On the other hand, suppose that Bob waits until $t_{i}+\tau$ so that Alice's $\left|\Psi_{c_{i}}\right\rangle$ enters his measurement device completely, and prepares the resent state following the measurement result. Then even though the form of the states will match perfectly, the resent state will reach Alice's detectors much later than it should when Bob uses the bypass mode, because the storage ring in channel $X$ of Alice's measurement device will delay the corresponding wave packet. This will make Alice aware that Bob is using the intercept mode too.

Alternatively, there can be another strategy where Bob simply sends all wave packets of his state simultaneously via one of the channels alone, e.g., through channel $X$ at time $t_{i}$ or through channel $Y$ at time $t_{i}+\tau$. But he will not be able to guarantee which of Alice's detectors will click with certainty.

Though here we have only analyzed the above few strategies as examples, the result is common. That is, in any strategy potentially existed, there will be a nonvanishing probability (let $\varepsilon$ denote the lower bound of the probability for all strategies) that Alice will find a mismatched result between the $\left|\Psi_{c_{i}}\right\rangle$ she sent and her measurement on the state she received from Bob, or the arrival time of Bob's state is different from what it should be in the bypass mode. This is because, as mentioned in section IV, the role of Bob in our protocol is actually the same as that of Eve in the KI QKD scheme [39]. If there is a strategy which is not bounded by the above result, then it can be utilized to fulfill a successful eavesdropping to the KI QKD scheme, which will conflict with the existing proof of the unconditional security of the scheme [39].

As a consequence, whenever Bob applies the intercept mode, Alice can distinguish it from the bypass mode with the probability $\varepsilon$. Then as described in step (6), by counting the number $n^{\prime}$ of the mismatched results, Alice can estimate the upper bound of the probability of Bob choosing the intercept mode as $f \sim n^{\prime} /(\varepsilon n)$. As she agrees to continue only when $f<1-d / n$, it is guaranteed that during the commit phase, Bob knows only $f n<n-d$ bits of $c$. Since feature (A) of the binary linear $(n, k, d)$-code $C$ ensures that the number of different codewords having $f n$ bits in common increases exponentially with $k$, the potential choices for $c$ are too much for Bob to determine whether $c$ belongs to the subset $C_{(0)}$ or $C_{(1)}$. Thus the amount of information Bob gained on the committed bit $b$ before the unveil phase can be made arbitrarily close to zero by increasing $k$.

Taking both Alice's and Bob's cases studied above into consideration, we can see that fixing $k / n$ and $d / n$ while increasing $n$ will then result in a protocol secure against both sides.

\section{B. More general proof: evading the MLC no-go theorem}

While it is hard to find a completely general proof like those for QKD [40] showing that the protocol is secure against all cheating strategies that may potentially exist, here it can be shown that our protocol is at least secure against the specific cheating strategy proposed in the MLC theorem.

\section{Essential of the no-go proof}

As pinpointed out in section 4 of [29], while there are many variations and extensions of the MLC no-go theorem [5]-28], their proofs all have the following common features.

(I) The reduced model. According to the no-go proofs, any QBC protocol can be reduced to the following model. Alice and Bob share a quantum state in a given Hilbert space. Each of them performs unitary transformations on the state in turns. All measurements are performed at the very end.

(II) The coding method. The quantum state corresponding to the committed bit $b$ has the form

$$
\left|\psi_{b}\right\rangle=\sum_{j} \lambda_{j}^{(b)}\left|e_{j}^{(b)}\right\rangle_{A} \otimes\left|f_{j}^{(b)}\right\rangle_{B} .
$$

Here the systems $A$ and $B$ are owned by Alice and Bob respectively.

(III) The concealing condition. To ensure that Bob's information on the committed bit is trivial before the unveil phase, any QBC protocol secure against Bob should satisfy

$$
\rho_{0}^{B} \simeq \rho_{1}^{B}
$$

where $\rho_{b}^{B} \equiv \operatorname{Tr}_{A}\left|\psi_{b}\right\rangle\left\langle\psi_{b}\right|$ is the reduced density matrix corresponding to Alice's committed bit $b$, obtained by tracing over system $A$ in equation (3). Note that in some presentation of the no-go proofs (e.g., [14, 16, 19, 26]), this feature was expressed using the trace distance or the fidelity instead of the reduced density matrices, while the meaning remains the same.

(IV) The cheating strategy. As long as equation (4) is satisfied, according to the Hughston-Jozsa-Wootters (HJW) theorem (a.k.a. the Uhlmann theorem, etc.) [41], there exists a local unitary transformation for Alice to map $\left\{\left|e_{j}^{(0)}\right\rangle_{A}\right\}$ into $\left\{\left|e_{j}^{(1)}\right\rangle_{A}\right\}$ successfully with a high probability [41]. Thus a dishonest Alice can unveil the state as either $\left|\psi_{0}\right\rangle$ or $\left|\psi_{1}\right\rangle$ at her will with a high probability to escape Bob's detection. Consequently, a concealing QBC protocol cannot be binding. 


\section{Equivalent model of our protocol}

Following the method of the MLC theorem to reduce our protocol into an equivalent form where Alice and Bob perform unitary transformations in turns on the quantum state they share, we can see that our commit protocol is essentially the following 3 -stage process.

(i) Alice prepares and sends Bob a system $\alpha$ containing $n$ qubits $\left|\Psi_{c_{i}}\right\rangle(i=1, \ldots, n)$.

(ii) Bob prepares an $n$-qubit system $\beta$ and an $n$-qutrit system $\gamma$. Then he performs an operation $U_{B}$ on the combined system $\beta \otimes \alpha \otimes \gamma$.

(iii) Bob sends system $\beta$ to Alice.

In this process, the initial state of each qubit in $\beta$ is chosen at Bob's choice, and kept secret from Alice. The system $\gamma$ is for storing the result of Bob's measurement on $\alpha$. The three orthogonal states of each qutrit in $\gamma$ are denoted as $|0\rangle,|1\rangle$ and $|2\rangle$, where $|0\rangle(|1\rangle)$ means that the measurement result of $\left|\Psi_{c_{i}}\right\rangle$ is $c_{i}=0\left(c_{i}=1\right)$, and $|2\rangle$ means that $\left|\Psi_{c_{i}}\right\rangle$ is not measured so that the result remains unknown. All the $n$ qutrits in system $\gamma$ are initialized in $|2\rangle$. The operation $U_{B}=u_{1} \otimes u_{2} \otimes \ldots \otimes u_{n}$ is defined by the mode that Bob chooses for each qubit in $\alpha$, where each $u_{i}(i=1, \ldots, n)$ is a 3 -particle operator acting on the $i$-th qubits/qutrit of $\beta, \alpha$ and $\gamma$. If Bob chooses the bypass mode for the $i$-th qubit of $\alpha$, then

$$
u_{i}=u_{b y p} \equiv P_{\beta \alpha} \otimes I_{\gamma} .
$$

Here $P_{\beta \alpha}$ is a 2-qubit permutation operator which interchanges the states of the $i$-th qubits of $\alpha$ and $\beta$, and $I_{\gamma}$ is the identity operator that keeps the $i$-th qutrit of $\gamma$ unchanged. Or if Bob chooses the intercept mode for the $i$-th qubit of $\alpha$, then

$$
u_{i}=u_{i n t} \equiv I_{\beta} \otimes\left(\left|\Psi_{0}\right\rangle_{\alpha}\left\langle\Psi_{0}\left|\otimes \mu_{\gamma}^{(0)}+\right| \Psi_{1}\right\rangle_{\alpha}\left\langle\Psi_{1}\right| \otimes \mu_{\gamma}^{(1)}\right) .
$$

Here $I_{\beta}$ is the identity operator on the $i$-th qubit of $\beta$, $\mu_{\gamma}^{(0)}\left(\mu_{\gamma}^{(1)}\right)$ is an unitary transformation on the $i$-th qutrit of $\gamma$ which can map the state $|2\rangle$ into $|0\rangle(|1\rangle)$. That is, applying $u_{\text {int }}$ is equivalent to measuring the $i$-th qubit of $\alpha$ and storing the result in the $i$-th qutrit of $\gamma$, while keeping the $i$-th qubit of $\beta$ unchanged. Among all the possible forms of the operation $U_{B}$, Bob chooses one of those that can pass the security check in step (6) of our original protocol in section IV (i.e., the number of $u_{\text {int }}$ in $U_{B}$ should be $f n<n-d$ ), and keeps his choice secret from Alice.

\section{Security against Alice's cheating}

Now we will show why the specific cheating strategy in the MLC theorem does not apply to our protocol. With the above equivalent description, we can see that after the commit phase, the density matrix of the systems shared between Alice and Bob is in the form $U_{B}\left(\rho^{\beta} \otimes \rho^{\alpha} \otimes \rho^{\gamma}\right) U_{B}^{\dagger}$, where $\rho^{\beta}, \rho^{\alpha}$ and $\rho^{\gamma}$ are the density matrices for the systems $\beta, \alpha$ and $\gamma$, respectively. Note that here the first $n$ qubits are now owned by Alice, i.e., they serve like the system $A$ in equation (3) even though system $\beta$ was prepared by Bob. Meanwhile, the rest qubits and qutrits now belong to Bob and serve like the system $B$ in equation (3), even though system $\alpha$ was prepared by Alice.

Let $\rho_{0}^{\alpha}\left(\rho_{1}^{\alpha}\right)$ denote the initial density matrix of system $\alpha$ prepared by Alice, that can unveil the committed bit as $b=0(b=1)$. According to the MLC theorem, now the goal for an dishonest Alice is to find a local unitary transformation $U_{A}$ acting on the $n$ qubits at her side alone, that can map $\rho_{0}^{B}$ into $\rho_{1}^{B}$. That is, $U_{A}$ should satisfy

$$
U_{A}\left[U_{B}\left(\rho^{\beta} \otimes \rho_{0}^{\alpha} \otimes \rho^{\gamma}\right) U_{B}^{\dagger}\right] U_{A}^{\dagger}=U_{B}\left(\rho^{\beta} \otimes \rho_{1}^{\alpha} \otimes \rho^{\gamma}\right) U_{B}^{\dagger} .
$$

Applying $U_{B}^{\dagger}(\ldots) U_{B}$ on both sides of this equation, we yield

$$
\left(U_{B}^{\dagger} U_{A} U_{B}\right)\left(\rho^{\beta} \otimes \rho_{0}^{\alpha} \otimes \rho^{\gamma}\right)\left(U_{B}^{\dagger} U_{A} U_{B}\right)^{\dagger}=\rho^{\beta} \otimes \rho_{1}^{\alpha} \otimes \rho^{\gamma} .
$$

Recall that $U_{B}$ was kept secret from Alice, thus she has to choose an $U_{A}$ which is independent of $U_{B}$. Meanwhile, the right side of equation (8) is independent of $U_{B}$ too. Therefore, equation (8) cannot be satisfied in general, unless $U_{A}$ always commutes with the specific $U_{B}$ 's that Bob may choose in the protocol. In this case $U_{B}^{\dagger} U_{A} U_{B}=$ $U_{A} U_{B}^{\dagger} U_{B}=U_{A}$, and equation (8) becomes

$$
U_{A}\left(\rho^{\beta} \otimes \rho_{0}^{\alpha} \otimes \rho^{\gamma}\right) U_{A}^{\dagger}=\rho^{\beta} \otimes \rho_{1}^{\alpha} \otimes \rho^{\gamma} .
$$

In many previous $\mathrm{QBC}$ protocols (e.g. [1, 2]) there is $\rho_{0}^{\alpha}=\rho_{1}^{\alpha}$. Then the HJW theorem guarantees that such a local $U_{A}$ exists, so that Alice can alter the value of her committed bit. This is why the cheating strategy in the MLC theorem is always successful in such protocols. However, as shown from equation (11), in our protocol $\left|\Psi_{0}\right\rangle$ and $\left|\Psi_{1}\right\rangle$ are orthogonal. Thus the state $\left|\psi_{c}\right\rangle \equiv\left|\Psi_{c_{1}}\right\rangle \otimes\left|\Psi_{c_{2}}\right\rangle \otimes \ldots \otimes\left|\Psi_{c_{i}}\right\rangle \otimes \ldots \otimes\left|\Psi_{c_{n}}\right\rangle$ corresponding to a specific codeword $c$ is orthogonal to the state corresponding to any other codeword. Consequently, our QBC protocol satisfies $\rho_{0}^{\alpha} \perp \rho_{1}^{\alpha}$, which is a crucial difference from previous insecure protocols. In this case the HJW theorem does not apply, making Alice unable to find an local transformation $U_{A}$ acting on her system (i.e., system $\beta$ ) alone while satisfying equation (9). For this reason, our protocol is immune to the specific cheating strategy proposed in the MLC no-go theorem.

\section{Security against Bob's cheating}

As the system $\alpha$ Alice sent to Bob in our QBC protocol satisfies $\rho_{0}^{\alpha} \perp \rho_{1}^{\alpha}$, at the first glance it seems that Bob can simply perform a measurement $M$ to project the state of $\alpha$ into the Hilbert spaces supported by $\rho_{b}^{\alpha}(b=0,1)$ and thus learn the value of Alice's committed $b$. But this is not true, because as shown above, the density matrix 
of the systems shared between Alice and Bob after the commit phase is $U_{B}\left(\rho^{\beta} \otimes \rho^{\alpha} \otimes \rho^{\gamma}\right) U_{B}^{\dagger}$. That is, Bob is required to perform the operation $U_{B}$ on system $\alpha$. This $U_{B}$ is incommutable with the measurement $M$ on $\alpha$ for distinguishing $\rho_{0}^{\alpha}$ and $\rho_{1}^{\alpha}$, since equation (5) indicates that $U_{B}$ contains permutation operators which act on not only system $\alpha$, but also other systems. Therefore, $U_{B}$ and $M$ cannot be both applied on $\alpha$. Bob can only choose one of them. The timing of the sending of the states in the protocol also prevents Bob from keeping system $\beta$ unsent until he receives the entire system $\alpha$. Thus he needs to decide on the fly which operation to apply. Once he applied $U_{B}$, then the state of $\alpha$ will be disturbed so that Bob will lose the chance to apply $M$ on it for decoding $b$.

Moreover, the difference between $U_{B}$ and $M$ is detectable to Alice. According to step (5) of our protocol, an $U_{B}$ is considered legitimate if it includes the operator $u_{\text {byp }}$ (i.e., Bob is using the bypass mode) for $(1-f) n>d$ times. On the contrary, because the minimal distance between the codewords is $d$, the number of $u_{b y p}$ in $M$ must be less than $d$. Otherwise as a basic property of the binary linear $(n, k, d)$-code, the number of the possible codewords having less than $n-d$ bits in common will be at the order of magnitude of $2^{k}$ so that such an $M$ can provide only trivial information on the value of Alice's committed $b$. But as elaborated in section V.A, the unconditional security of the KI QKD scheme 39] ensures that, for each of Alice's state $\left|\Psi_{c_{i}}\right\rangle$ which Bob applies the intercept mode, his resent state can equal to $\left|\Psi_{c_{i}}\right\rangle$ with the probability $1-\varepsilon$ only, where there is always $\varepsilon>0$ no matter which resend strategy Bob uses. When the number of the applied bypass mode is less than $d$, Alice will find that the number of mismatched result between her received state and the original $\left|\Psi_{c_{i}}\right\rangle$ she sent will be $n^{\prime}>\varepsilon(n-d)$. If she takes $f \equiv n^{\prime} /(\varepsilon n)$ as stated in step (6) of the protocol, then she will find that $f>1-d / n$. Thus she knows that Bob was attempting to apply the operation $M$ instead of a legitimate $U_{B}$.

Namely, while Alice's committed state satisfies $\rho_{0}^{\alpha} \perp$ $\rho_{1}^{\alpha}$ so that it is distinguishable to Bob, Alice's security check in the protocol requires Bob to perform an operation $U_{B}$, which is incommutable with the operation $M$ for distinguishing $\rho_{0}^{\alpha}$ and $\rho_{1}^{\alpha}$. The protocol thus becomes concealing against Bob.

\section{FEASIBILITY}

In the above we focused only on the theoretical possibility of evading the MLC no-go theorem. But we can see from FIG. 2 that our protocol is also very feasible, as the required experimental technology is already available today [42].

Also, as the secret random sending time is no longer required, the commit phase will take less time than that of our previous protocol [29], and the total number of photons that Bob needs to send in the intercept mode will be significantly reduced. More rigorously, suppose that the minimal time for Bob to shift between the intercept and bypass modes is $\Delta$. When both protocols choose the same $n$ as the length of the codewords, our previous protocol [29] requires Bob to send $s f(s>>n$. Note that $f$ was denoted as $\alpha$ in [29]) photons in total, and the duration time of the commit phase is $s \Delta$. But in the current protocol, the photon number is reduced to $n f$, and the duration time is $n \Delta$. As it was suggested in section 3 of [29] that a typical choice of the parameters is $s / n=10$, we can see that the current protocol can be 10 times more efficient than our previous one [29].

There is another advantage of removing the need to keep the sending time secret at first. That is, now Alice and Bob can determine the binary linear $(n, k, d)$-code $C$ and decide on the sending time of the states $\left|\Psi_{c_{i}}\right\rangle$ $(i=1, \ldots, n)$ beforehand, so that no more classical communication is needed at all during the commit phase, unless one of them finds the other participant cheating and announces to abort. Therefore, it not only reduces the communication cost significantly, but also makes it easier for security analysis and comparison with the MLC theorem, which was generally deduced in a theoretical model where classical communication is not presented directly.

Nevertheless, under practical settings, some more security checks should be added against technical attacks. Especially, the physical systems implementing the states may actually have other degrees of freedom, which leave rooms for Alice's cheating. For example, she may send photons with certain polarization or frequency, so that she can distinguish them from the photons Bob sends in the intercept mode. In this case, Bob and Alice should discuss at the beginning of the protocol, to limit these degrees of freedom to a single mode. In step (5) when Bob chooses the intercept mode, he should also measure occasionally these degrees of freedom of some of Alice's photons, instead of performing the measurement in the original step (5). Then if Alice wants to send distinguishable photons with a high probability so that they are sufficient for her cheating, she will inevitably be detected.

Moreover, when Bob applies the bypass mode, he should add phase shifters to both channels $X$ and $Y$ to introduce the same phase shift in both channels so that an honest Alice will not be affected (to be explained in the appendix), while the amount of this phase shift is randomly chosen and kept secret from Alice, so that the counterfactual attack described in the appendix can be defeated.

Note that all these technical attacks and the corresponding modifications are due to the imperfection of the physical systems implementing the protocol. They should not be messed with the theoretical possibility of evading the MLC no-go theorem. 


\section{SUMMARY AND DISCUSSIONS}

In conclusion, inspired by the KI simplified version 39] of the GV QKD scheme 38], we improved our previously proposed QBC protocol [29], and proved that it is immune to the specific cheating strategy used in the MLC no-go theorem of unconditionally secure QBC. The key reason is that the density matrices of Alice's states corresponding to the committed values 0 and 1 are orthogonal to each other, making her unable to find the local unitary transformation for the cheating by using the HJW theorem. Meanwhile, the protocol remains concealing against Bob because he is required to perform another operation, which is incommutable with the measurement for distinguishing the density matrices.

However, there may potentially exist innumerous cheating strategies other than the one in the MLC theorem. It is natural to ask whether our protocol can be unconditionally secure against all these strategies. A rigorous evaluation of the upper bound of the cheating probability is also needed. For example, our protocol involves a probability $\varepsilon$ which denotes the lower bound of the average probability for Bob's resent state in step (5) to be caught in Alice's check. The exact value of $\varepsilon$ should be determined by the rigorous quantitatively security analysis of the KI QKD scheme. Unfortunately, such a value was not yet provided in the literature [39]. In turns, the parameters $k$ and $d$ in the binary linear $(n, k, d)$-code $C$ used in our protocol need to be chosen according to $\varepsilon$. Therefore it remains unknown whether the cheater can have a nontrivial probability of success if these parameters are improperly chosen. We would like to leave these questions open for future researches.

Though the current QBC protocol and the one in 29] have similarities in many ways, the underlying origins of their security against Bob are somewhat different. While both protocols are immune to Bob's cheating because they are based on unconditionally secure QKD schemes, as pointed out in [39], the GV QKD scheme can actually be viewed as utilizing three orthogonal states - two photon states and one vacuum state. Its security is provided by the random sending time of the photons. On the contrary, the KI QKD scheme does not require the vacuum state and the secret sending time. The security is guaranteed by the fact that the eavesdropper cannot fake the states with certainty owe to the use of the asymmetric beam splitters. Similarly, the security of the QBC protocol in [29] against Bob is based on Alice's random sending time that remains secret before the last step of the commit phase, while in our current QBC proposal, it is because Bob cannot fake the states with certainty when he runs the intercept mode. Therefore our current protocol is more than merely a simplification on the presentation.

The work was supported in part by the NSF of China, the NSF of Guangdong province, and the Foundation of Zhongshan University Advanced Research Center.

\section{Appendix A: Defeating the counterfactual attack}

As we mentioned in Sec. 6, under practical settings our QBC protocol may need some modifications against technical attacks. Here we give such an example.

Recently a cheating strategy against counterfactual QKD protocols [43, 44] was proposed [45]. Unlike general intercept-resend attacks in which measurements are performed on the quantum states carrying the secret information, in this strategy the cheater makes use of quantum counterfactual effect to detect the working modes of the devices of other participants. Thus it was named "the counterfactual attack" [45]. Here we will skip how it applies to QKD protocols, while focus only on its impact on our QBC protocol.

FIG. 3 illustrates the apparatus for the attack [45]. The core is a "fictitious" beam splitter (FBS) which has the following functions.

(f1) Any photon hitting the FBS from path $c$ will be reflected with certainty.

(f2) When the paths $a$ and $b$ are adjusted correctly, two wave packets coming from paths $a$ and $b$, respectively, will interfere and combine together, and enter path $c$ with certainty.

(f3) Any photon hitting the FBS from path $a$ alone will pass through the FBS and enter path $d$ with certainty.

An ideal FBS that can realize these functions faithfully does not exist in principle. Thus it is called "fictitious". For example, devices with the functions (f2) and (f3) may not accomplish the function (f1) perfectly, i.e., a photon coming from path $c$ could pass the devices with a nontrivial probability, making the attack detectable. However, FBS can be implemented approximately by using an infinite number of ordinary BS 44, 45]. In practice, the number of BS involved in the implementation has to be finite. But if the deviation from an ideal FBS is too small to be detected within the capability of available technology, then the attack could become a real threat.

Suppose that an ideal FBS is available to a dishonest Alice in our QBC protocol. When she is supposed to send Bob a state in step (4), she runs both the FBS system in FIG. 3 and the apparatus in the honest protocol (i.e., the one shown in FIG. 2) simultaneously in parallel, with path $b$ of the FBS system connecting to both the input and output of Bob's channel $X$ (or both the input and output of Bob's channel $Y$ ). The apparatus in FIG. 2 works as usual so that the protocol can be executed as if she is honest, while the FBS system serves as a probe to detect Bob's mode. According to the function (f2) of the FBS, whenever Bob applies the bypass mode in step (5), the wave packets of a photon Alice sent to the FBS will be returned from both paths $a$ and $b$ so that the detector $D_{c}$ will click with certainty. On the other hand, whenever Bob applies the intercept mode, an ideal FBS can guarantee that $D_{c}$ will never click as path $b$ is actually blocked. Therefore Alice can learn Bob's mode unambiguously. Since Bob does not know the state $\left|\Psi_{c_{i}}\right\rangle$ Alice sends when he applies the bypass mode, Alice can 


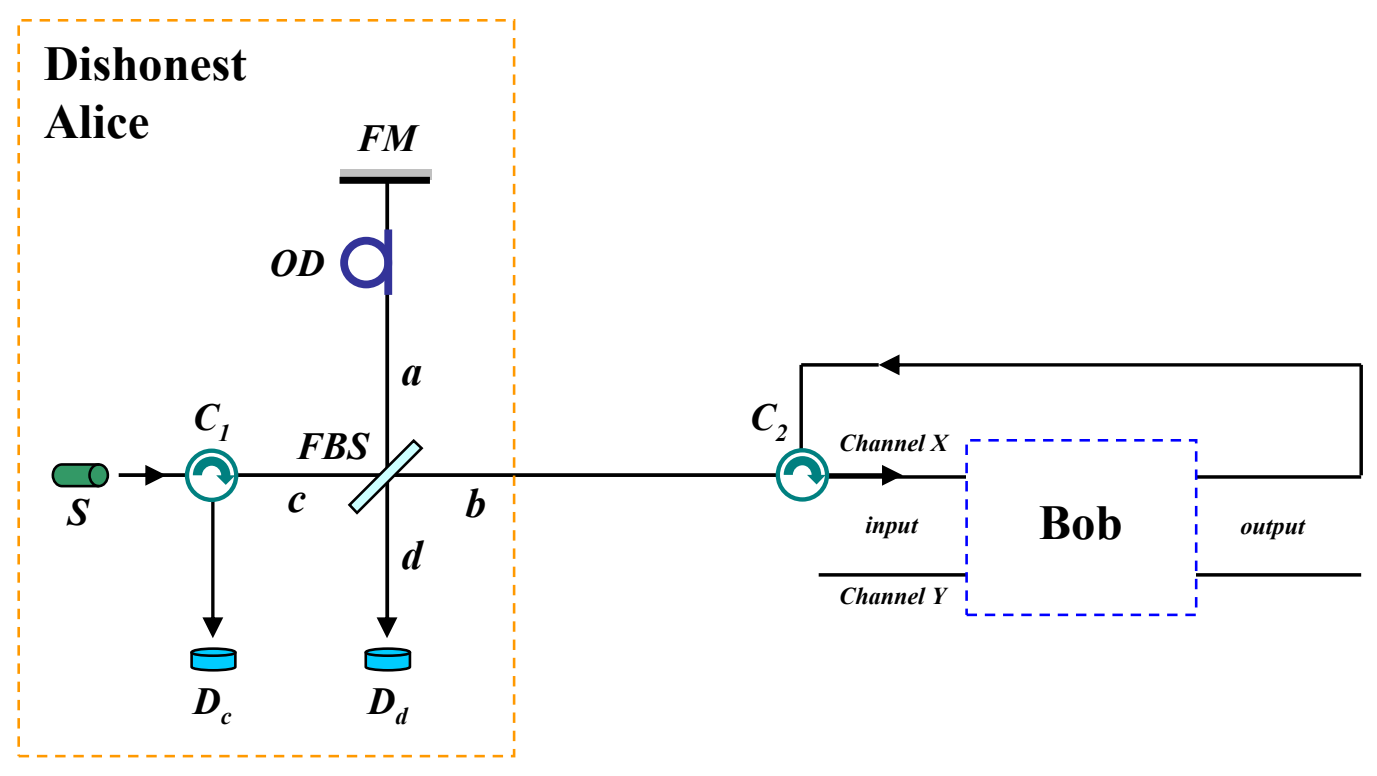

FIG. 3: Diagram of the apparatus for Alice's counterfactual attack. A single-photon pulse produced by the source $S$ passes through the optical circulator $C_{1}$ and hits the "fictitious" beam splitter (FBS) along path $c$. Path $a$ is adjusted by the optical delay $O D$, followed by a Faraday mirror $F M$. Any photon coming from path $c$ from the right to the left will be detected by the detector $D_{c}$, while the detector $D_{d}$ detects any photon coming from path $d$. Path $b$ is connected to both the input and output of Bob's channel $X$ (or both the input and output of Bob's channel $Y$ ) via the optical circulator $C_{2}$.

lie about the value of the corresponding $c_{i}$ freely, thus alters her committed $b$ in the unveil phase.

Nevertheless, it is easy to defeat this counterfactual attack. As pointed out in Ref. [45], Bob's randomizing the optical length of path $b$ is sufficient to destroy the interference effect in the FBS system. Therefore in our protocol, Bob can simply add extra phase shifters (other than the one shown in FIG. 2) to both channels $X$ and $Y$ when he applies the bypass mode, to introduce the same phase shift $e^{i \theta}$ in both channels, where the value of $\theta$ is randomly chosen and kept secret from Alice, and can vary for different $\left|\Psi_{c_{i}}\right\rangle$. In this case, after passing Bob's apparatus, Alice's initial states $\left|\Psi_{0}\right\rangle$ and $\left|\Psi_{1}\right\rangle$ will become, respectively,

$$
\begin{aligned}
\left|\Psi_{0}\right\rangle \rightarrow & \left|\Psi_{0}^{\prime}\right\rangle \equiv \sqrt{T}\left(e^{i \theta}|0\rangle_{X}\right)\left(e^{i \theta}|1\rangle_{Y}\right) \\
& -i \sqrt{R}\left(e^{i \theta}|1\rangle_{X}\right)\left(e^{i \theta}|0\rangle_{Y}\right), \\
\left|\Psi_{1}\right\rangle \rightarrow & \left|\Psi_{1}^{\prime}\right\rangle \equiv \sqrt{T}\left(e^{i \theta}|1\rangle_{X}\right)\left(e^{i \theta}|0\rangle_{Y}\right) \\
& -i \sqrt{R}\left(e^{i \theta}|0\rangle_{X}\right)\left(e^{i \theta}|1\rangle_{Y}\right) .
\end{aligned}
$$

We can see that $\left|\Psi_{0}^{\prime}\right\rangle\left(\left|\Psi_{1}^{\prime}\right\rangle\right)$ differs from $\left|\Psi_{0}\right\rangle\left(\left|\Psi_{1}\right\rangle\right)$ merely by a global factor $e^{i 2 \theta}$. It is well known that such a global factor is not detectable. In fact, in our case the interference pattern of the two wave packets meeting at the beam splitter of Alice's measurement device is determined by their relative phase difference. No change will be detected when they both receive a phase shift $e^{i \theta}$. Thus an honest Alice who uses the original apparatus described in FIG. 2 will still detect $\left|\Psi_{0}^{\prime}\right\rangle\left(\left|\Psi_{1}^{\prime}\right\rangle\right)$ as $\left|\Psi_{0}\right\rangle$ $\left(\left|\Psi_{1}\right\rangle\right)$, even though she does not know the value of $\theta$. On the other hand, if Alice wants to apply the above counterfactual attack, without knowing $\theta$ she cannot know how to adjust path $a$ to ensure $D_{c}$ in FIG. 3 clicking with certainty. Consequently, there will be times that Alice does not know which mode Bob is running. Then the number of $c_{i}$ 's that she can alter will be limited, which is insufficient to change the committed $b$ as long as the value of $d / n$ in our QBC protocol is properly chosen.
[1] Bennett, C.H., Brassard, G.: Quantum cryptography: Public key distribution and coin tossing. In: Proceedings of IEEE International Conference on Computers, Systems, and Signal Processing, pp. 175. IEEE, New York (1984)

[2] Brassard, G., Crépeau, C., Jozsa, R., Langlois, D.: A quantum bit commitment scheme provably unbreakable by both parties. In Proceedings of 34th Annual IEEE Symposium on Foundations of Computer Science, pp. 362. IEEE, Los Alamitos (1993)

[3] Yao, A.C.C.: Security of quantum protocols against coherent measurements. In: Proceedings of 26th Sympo- 
sium on the Theory of Computing, pp. 67. ACM, New York (1995)

[4] Kilian, J.: Founding crytpography on oblivious transfer. In: Proceedings of 1988 ACM Annual Symposium on Theory of Computing, pp. 20. ACM, New York (1988)

[5] Mayers, D.: The trouble with quantum bit commitment. e-print. quant-ph/9603015v3 (1996)

[6] Mayers, D.: Unconditionally secure quantum bit commitment is impossible. Phys. Rev. Lett. 78, 3414 (1997)

[7] Lo, H. -K., Chau, H. F.: Is quantum bit commitment really possible? Phys. Rev. Lett. 78, 3410 (1997)

[8] Crépeau, C.: What is going on with quantum bit commitment? In: Proceedings of Pragocrypt '96: 1st International Conference on the Theory and Applications of Cryptology. Czech Technical University Publishing House, Prague (1996)

[9] Chau, H. F., Lo, H. -K.: Making an empty promise with a quantum computer. Fortsch. Phys. 46, 507 (1998)

[10] Lo, H. -K., Chau, H. F.: Why quantum bit commitment and ideal quantum coin tossing are impossible. Physica D 120, 177 (1998)

[11] Bub, J.: The quantum bit commitment theorem. Found. Phys. 31, 735 (2001)

[12] Brassard, G., Crépeau, C., Mayers, D., Salvail, L.: A brief review on the impossibility of quantum bit commitment. e-print. quant-ph/9712023v1 (1997)

[13] Brassard, G., Crépeau, C., Mayers, D., Salvail, L.: Defeating classical bit commitments with a quantum computer. e-print. quant-ph/9806031 v1 (1998)

[14] Spekkens, R.W., Rudolph, T.: Degrees of concealment and bindingness in quantum bit commitment protocols. Phys. Rev. A 65, 012310 (2001)

[15] Spekkens, R.W., Rudolph, T.: Optimization of coherent attacks in generalizations of the BB84 quantum bit commitment protocol. Quantum Inf. Comput. 2, 66 (2002). e-print. quant-ph/0107042 2 (2001)

[16] Chailloux, A., Kerenidis, I.: Optimal bounds for quantum bit commitment. e-print. arXiv:1102.1678v1 (2011)

[17] D'Ariano, G.M.: The quantum bit commitment: A finite open system approach for a complete classification of protocols. e-print. quant-ph/0209149v1 (2002)

[18] D'Ariano, G.M.: The quantum bit commitment: A complete classification of protocols (Shortened version of quant-ph/0209149). In: Proceedings of QCM\&C. Rinton press, Boston. Also available as. e-print. quant-ph/0209150v1 (2002)

[19] D'Ariano, G.M., Kretschmann, D., Schlingemann, D., Werner, R.F.: Reexamination of quantum bit commitment: The possible and the impossible. Phys. Rev. A 76, 032328 (2007). e-print. quant-ph/0605224 v2 (2006)

[20] Chiribella, G., D'Ariano, G.M., Perinotti, P., Schlingemann, D.M., Werner, R.F.: A short impossibility proof of quantum bit commitment. Phys. Lett. A 377, 1076 (2013). e-print. arXiv:0905.3801 v1 (2009)

[21] Mayers, D.: Superselection rules in quantum cryptography. e-print. quant-ph/0212159v2 (2002)

[22] Kitaev, A., Mayers, D., Preskill, J.: Superselection rules and quantum protocols. Phys. Rev. A 69, 052326 (2004)

[23] Halvorson, H.: Remote preparation of arbitrary ensembles and quantum bit commitment. J. Math. Phys. 45, 4920 (2004). e-print. quant-ph/0310001 v2 (2003)

[24] Cheung, C.-Y.: Secret parameters in quantum bit commitment. In: Proceedings of ERATO Conference on Quantum Information Science 2005. Tokyo. Also avail- able as. e-print. quant-ph/0508180v2 (2005)

[25] Cheung, C.-Y.: Insecurity of quantum bit commitment with secret parameters. e-print. quant-ph/0601206v1 (2006)

[26] Magnin, L., Magniez, F., Leverrier, A., Cerf, N.J.: Strong no-go theorem for Gaussian quantum bit commitment. Phys. Rev. A 81, 010302(R) (2010). e-print. arXiv:0905.3419v2 (2009)

[27] Chiribella, G., D'Ariano, G.M., Perinotti, P.: Probabilistic theories with purification. Phys. Rev. A 81, 062348 (2010). e-print. arXiv:0908.1583v5 (2009)

[28] Li, Q., Li, C.Q., Long, D.-Y., Chan, W.H., Wu, C.-H.: On the impossibility of non-static quantum bit commitment between two parties. Quantum Inf. Process. 11, 519 (2012). e-print. arXiv:1101.5684v1 (2011)

[29] He, G.P.: Quantum key distribution based on orthogonal states allows secure quantum bit commitment. J. Phys. A Math. Theor. 44, 445305 (2011)

[30] He, G.P.: Secure quantum bit commitment against empty promises. Phys. Rev. A 74, 022332 (2006). (Extended version of [31] with refrained claim on the security level)

[31] He, G.P.: Quantum bit commitment using entangled states. e-print. quant-ph/0303107 (2003)

[32] He, G.P.: Secure quantum bit commitment against empty promises. II. The density matrix. e-print. arXiv:1307.7318 (2013)

[33] Yuen, H.P.: An unconditionally secure quantum bit commitment protocol. e-print. arXiv:1212.0938 v (2012)

[34] Srikanth, R.: Quantum bit commitment with a composite evidence. Phys. Scr. 70, 343 (2004).

[35] Hardy, L., Kent, A.: Cheat sensitive quantum bit commitment. Phys. Rev. Lett. 92, 157901 (2004)

[36] Shimizu, K., Fukasaka, H., Tamaki, K., Imoto, N.: Cheat-sensitive commitment of a classical bit coded in a block of $m \times n$ round-trip qubits. Phys. Rev. A 84, 022308 (2011)

[37] Li, Y.-B., Wen, Q.-Y., Li, Z.-C., Qin, S.-J., Yang, Y.-T.: Cheat sensitive quantum bit commitment via pre- and post-selected quantum states. Quantum Inf. Process. 13, 141 (2014)

[38] Goldenberg, L., Vaidman, L.: Quantum cryptography based on orthogonal states. Phys. Rev. Lett. 75, 1239 (1995)

[39] Koashi, M., Imoto, N.: Quantum cryptography based on split transmission of one-bit information in two steps. Phys. Rev. Lett. 79, 2383 (1997)

[40] Shor, P. W., Preskill, J.: Simple proof of security of the BB84 quantum key distribution protocol. Phys. Rev. Lett. 85, 441 (2000)

[41] Hughston, L. P., Jozsa, R., Wootters, W. K.: A complete classification of quantum ensembles having a given density matrix. Phys. Lett. A 183, 14 (1993)

[42] Avella, A., Brida, G., Degiovanni, I. P., Genovese, M., Gramegna, M., Traina, P.: Experimental quantumcryptography scheme based on orthogonal states. Phys. Rev. A 82, 062309 (2010)

[43] Noh, T. -G.: Counterfactual quantum cryptography. Phys. Rev. Lett. 103, 230501 (2009)

[44] Sun, Y., Wen, Q. -Y.: Counterfactual quantum key distribution with high efficiency. Phys. Rev. A 82, 052318 (2010)

[45] Zhang, S., Wnang, J., Tang, C. J.: Counterfactual attack on counterfactual quantum key distribution. EPL 
98, $30012(2012)$ 